\title{
e-Migrinter
}

15 | 2017

Migrinter a trente ans : analyses et portraits

\section{Des expositions hors les murs : retour sur le projet Démineurs}

Sarah Przybyl et Fred Soupa

\section{CpenEdition}

Journals

Édition électronique

URL : https://journals.openedition.org/e-migrinter/832

DOI : $10.4000 /$ e-migrinter.832

ISSN : 1961-9685

Éditeur

UMR 7301 - Migrinter

Référence électronique

Sarah Przybyl et Fred Soupa, "Des expositions hors les murs : retour sur le projet Démineurs », eMigrinter [En ligne], 15 | 2017, mis en ligne le, consulté le 20 mai 2021. URL : http://

journals.openedition.org/e-migrinter/832 ; DOI : https://doi.org/10.4000/e-migrinter.832

Ce document a été généré automatiquement le 20 mai 2021.

Tous droits réservés 


\title{
Des expositions hors les murs : retour sur le projet Démineurs
}

\author{
Sarah Przybyl et Fred Soupa
}

1 Démineurs est un projet audiovisuel visant la réalisation d'ateliers d'expression dans des structures spécialisées dans l'accueil des mineurs isolés étrangers ${ }^{1}$. Le dispositif propose à des mineurs et des adultes volontaires un espace de parole où ils sont libres de traiter d'un thème de leur choix (différences culturelles, réalité de l'action éducative, parcours migratoire, etc.). Après une mise en son et en images de leur récit, le travail de montage délivre ces témoignages sous la forme de court-métrages. L'objectif du projet Démineurs est de donner la parole à des mineurs sur un sujet libre en dehors de toute injonction institutionnelle.

2 La richesse du projet réside dans la diversité des pays de provenance des participants. En effet, les jeunes qui ont souhaité s'exprimer dans le cadre des ateliers viennent d'Afghanistan, de Guinée-Conakry, du Pakistan, de République démocratique du Congo, du Mali, d'Angola, de Côte d'Ivoire, de Tunisie ou du Bengladesh. Ils (et elles) ont entre 13 et 17 ans, parlent plusieurs langues, mais ne les écrivent pas toujours. Certains ont été scolarisés, d'autres jamais. Tous ont parcourus des milliers de kilomètres dans des conditions et pour des raisons très différentes. 
Photo $\mathrm{n}^{\circ} 1$ : Les langues parlées par Téjinder (16 ans, Inde)

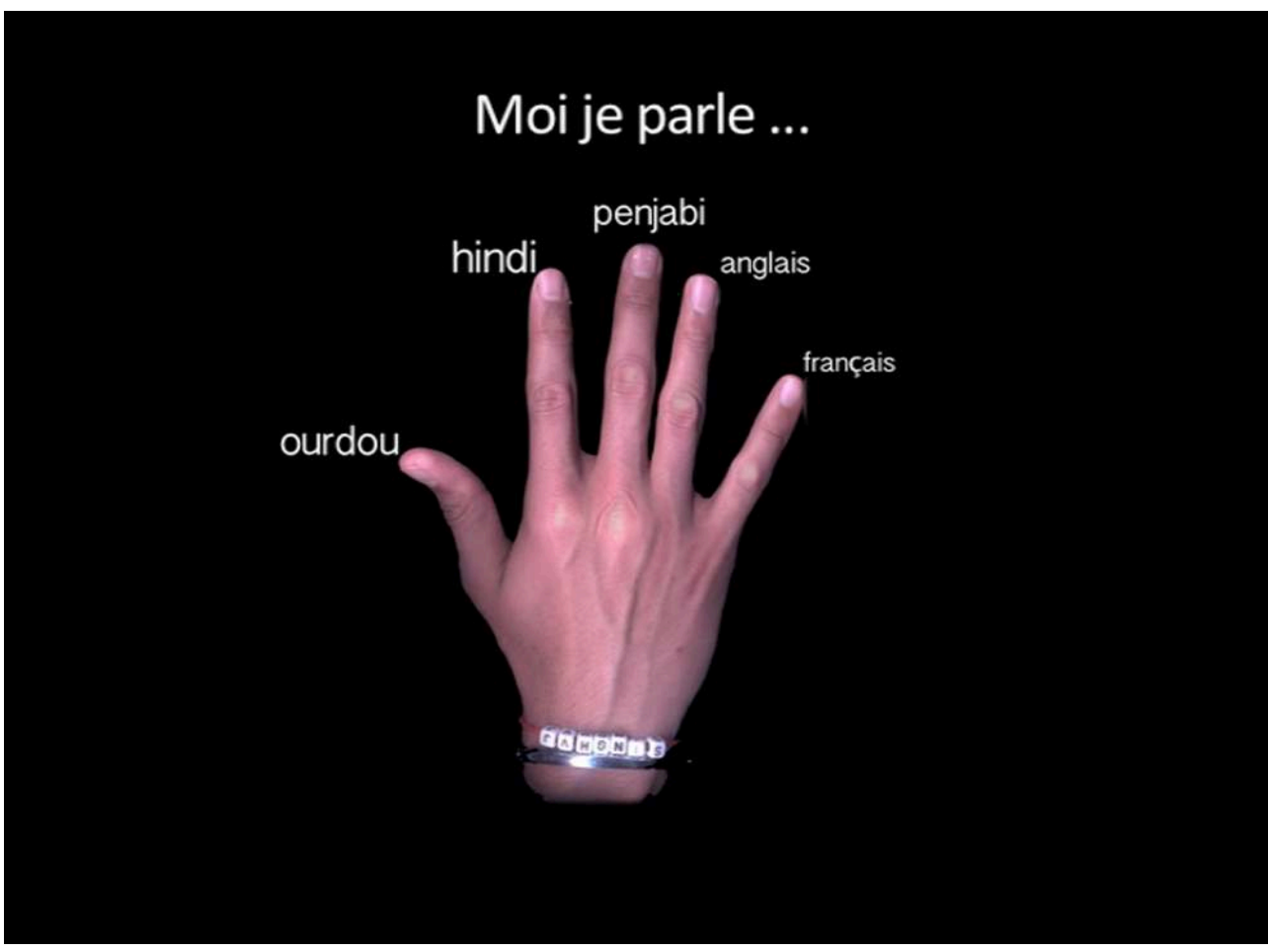

Prise de vue : Sarah Przybyl et Fred Soupa.

3 Aujourd'hui, il est impossible de passer sous silence l'importance des politiques en matière de prise en charge des mineurs tant les évolutions législatives ont redessiné les contours de ce projet. Démineurs s'inscrit dans un contexte où, pour bénéficier d'une protection, les jeunes doivent prouver leur minorité et leur isolement lors d'une évaluation dite sociale. Cette procédure de vérification de la situation est régie au plan national par une circulaire du ministère de la Justice datant de mai 2013. Différentes rubriques doivent être renseignées puis envoyées au Conseil départemental où se trouve le mineur. Tel que décrit dans la circulaire, ce faisceau d'indices aide les autorités à se prononcer sur l'admissibilité du mineur par les services de l'Aide sociale à l'enfance (Bailleul et Senovilla Hernández, 2016 ; Ministère de la justice, 2013(a) et (b)).

4 Alors que leur situation d'isolement et leur minorité en font des enfants en danger à protéger, les préoccupations croissantes pour la gestion des migrations internationales ont fait évoluer le regard porté sur ces jeunes. Suspicion, contrôle, mise en doute du récit, etc. dessinent un parcours d'accès à la protection de plus en plus chaotique. Une fois pris en charge, la parole des jeunes reste un outil central du parcours de protection. Rendez-vous avec les représentants des institutions, échanges avec le personnel éducatif, apprentissage de la langue française, etc. constituent autant de moments quotidiens où l'expression orale des jeunes est mobilisée comme un support de l'action éducative (Przybyl, 2016).

5 Dans un contexte où la parole des mineurs est mise à rude épreuve, régulièrement sollicitée et peut faire l'objet d'un contrôle constant, le temps d'un atelier, Démineurs ouvre et propose un espace d'expression inédit. Plusieurs lieux d'accueil en France menant de missions différentes dans le cadre du parcours de prise en charge des mineurs ont rendu possible cette aventure et ces rencontres. Dans des lieux d'accueil 
d'urgence ou dans des Maisons d'enfants à caractère social (MECS), les paroles des jeunes recueillies se situent à différents moments de la prise en charge.

6 À Neuilly-sur-Marne (association Devenir les Gavroches), à Saverdun en Ariège (Institut protestant de Saverdun), à Taverny (Lieu d'accueil et d'orientation de la Croix-Rouge française) et à Paris (Service d'accueil de jour pour mineurs isolés étrangers de la CroixRouge française), nous avons eu la possibilité de mener plusieurs ateliers en collaboration avec les équipes éducatives des lieux spécialisés dans l'accueil des mineurs isolés étrangers.

Photo $\mathrm{n}^{\circ} 2$ : Lieu d'accueil et d'orientation à Taverny

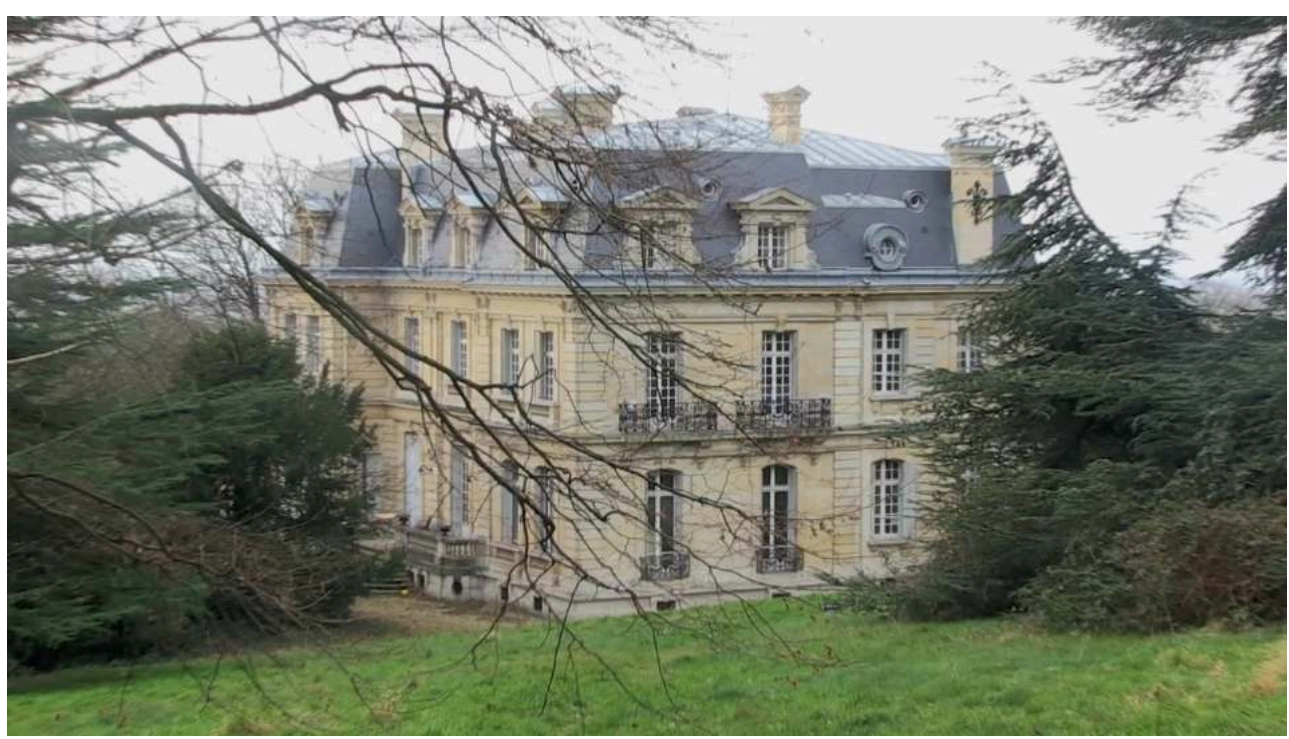

Prise de vue : Sarah Przybyl et Fred Soupa.

7 Les sujets choisis par ces adolescents reflètent la diversité des préoccupations qui les animent lors de leur participation aux ateliers. Leur histoire migratoire immédiate, les différences culturelles et sociales entre leur pays d'origine et la France, leur quotidien, leurs rêves d'avenir ou encore la dénonciation de certaines réalités sont autant de thématiques librement choisies et abordées dans les récits. Au total, près de 35 courtmétrages ont été réalisés avec des jeunes pris en charge dans les structures ayant accueilli le projet Démineurs. En résulte des petits films documentaires d'animation, personnels ou co-construits. Nourris de récits pudiques ou frontaux, de sons et d'images émanant exclusivement d'eux, ces témoignages créatifs rendent audibles et visibles les réalités, le parcours physique et intérieur, et les questionnements de ces adolescents venus d'ailleurs. Sans exhaustivité, ces témoignages reflètent surtout leur désir d'expression.

8 À l'occasion de la réalisation d'ateliers au sein du Lieu d'accueil et d'orientation (LAO) à Taverny, nous avons proposé aux professionnels de se prêter au même exercice que les jeunes. Dans le cadre d'une collaboration avec le dispositif Passeurs d'images autour de la thématique des frontières, plusieurs adultes du lieu ont accepté de livrer leur interprétation de ce thème à travers le traitement de sujets de leur choix. Éducateurs spécialisés, chef de service, médecins, professeur de français langue étrangère, professionnel technique, socio-esthéticienne posent un regard sur leur pratique professionnelle auprès des mineurs isolés étrangers. La parole des acteurs de terrain 
est aussi l'occasion de voir comment, au-delà du rapport professionnel qu'ils entretiennent, l'action auprès de ces jeunes fait écho aux parcours plus personnels de certains de ces intervenants.

Outre la dimension artistique et créative, le projet Démineurs s'inscrit dans un volet scientifique. En effet, en plus des ateliers de création, le travail de recherche mené par Sarah Przybyl dans plusieurs lieux d'accueil en France (dont des structures participants au projet) permet d'éclairer autrement les paroles des jeunes. Son approche par la géographie sociale permet d'apprécier plus largement les parcours migratoires et de protection des mineurs isolés étrangers. Le choix de mobiliser l'outil audiovisuel dans une démarche scientifique s'inscrit dans la lignée d'innovations méthodologiques qui ne cessent d'émerger en géographie et plus largement en sciences humaines et sociales. La rencontre entre recherche et création née du projet Démineurs révèle tout l'intérêt pour les scientifiques au contact de terrains sensibles d'envisager des nouvelles pistes pour recueillir la parole des populations enquêtées. En effet, les outils artistiques permettent de proposer aux enquêtés d'autres supports pour se raconter et leur laissent une certaine liberté au moment de parler d'eux. Loin du cadre d'un entretien semi-directif, les ateliers audiovisuels ont permis aux mineurs de travailler leur récit pendant plusieurs jours, de s'assurer du choix des mots employés et d'échanger avec les autres participants. En coulisse, le processus d'écriture, de mise en mots et en images crée un espace propice au dialogue informel et à l'échange spontané. Outre la richesse de chaque court-métrage, ce sont ces instants de création qui ont permis d'élaborer les premiers contacts avec les mineurs et préparer avec eux les conditions propices pour des échanges plus approfondis.

10 Aujourd'hui, à mesure que la question des mineurs isolés étrangers devient un sujet de société celle-ci fait l'objet de productions académiques, institutionnelles, associatives et artistiques multiples. Pour proposer au public de mieux cerner les enjeux de cette population, les différents récits récoltés lors du projet Démineurs sont aujourd'hui réunis dans une exposition. Composée de panneaux explicatifs relatant la genèse du projet et proposant la visualisation de plusieurs court-métrages, cette exposition ambitionne également de renseigner le public sur les évolutions légales et les réalités vécues par cette population à l'échelle nationale. Cette restitution révèle le portrait individuel de ces jeunes venus d'ailleurs et dévoile le quotidien méconnu des travailleurs sociaux dans des lieux d'accueil. Outre la possibilité de voir et entendre les témoignages de mineurs et de professionnels, de découvrir la démarche de création et les productions graphiques des participants, l'exposition est accompagnée de projections et de conférences animées par Fred Soupa et Sarah Przybyl. Ces temps d'échange sont l'occasion d'initier des débats collectifs, de susciter des réactions et parfois, plus simplement, des émotions. Outil de sensibilisation à la thématique, découverte des conditions de vie de ces jeunes, appréhension d'enjeux politiques, Démineurs ouvre la porte au dialogue autour d'un sujet plus que jamais d'actualité.

Pour en savoir plus sur l'exposition : www.demineurs.com 
Photo $n^{\circ} 3$ : Dessins réalisés par les participants lors des ateliers
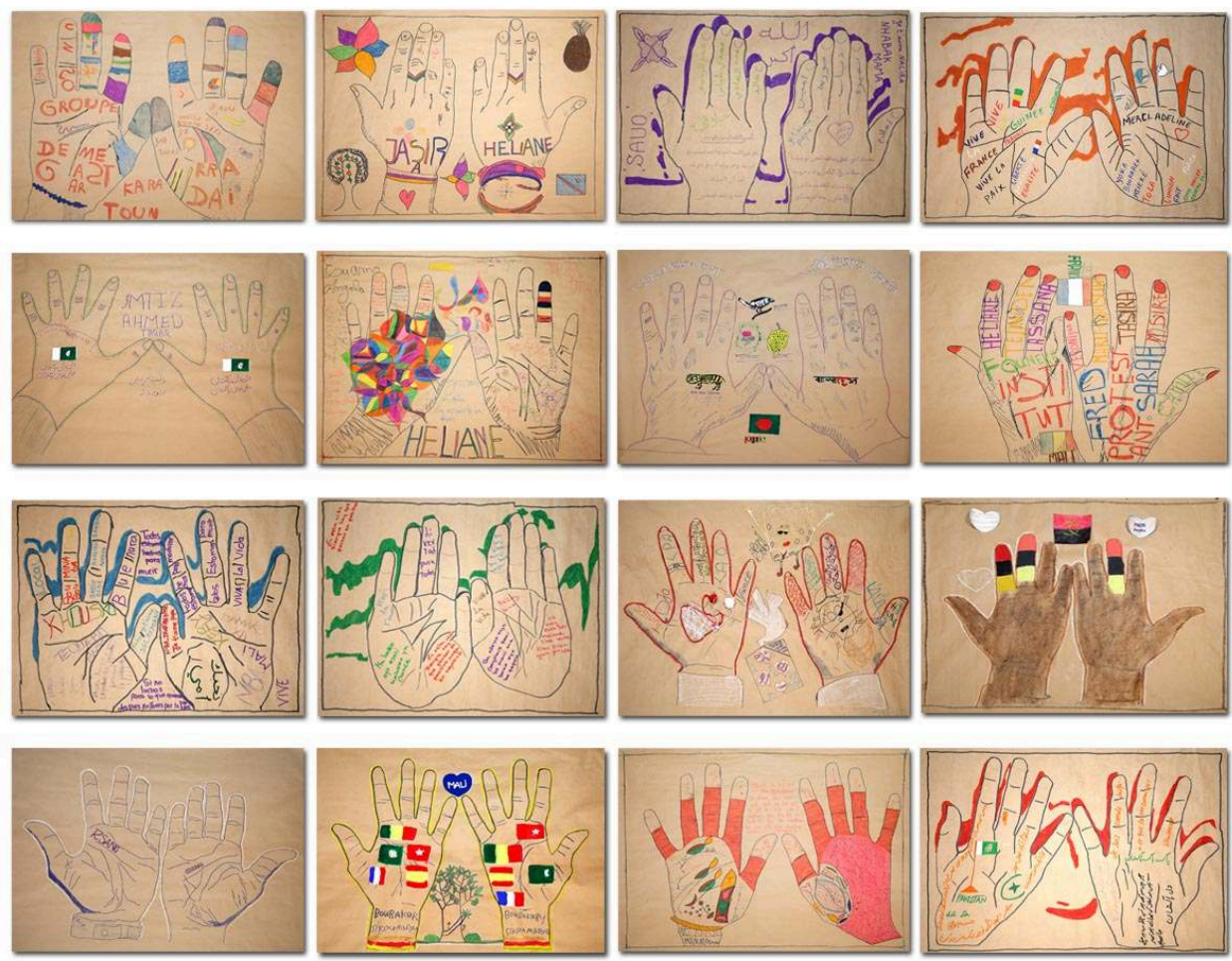

Prise de vue : Sarah Przybyl et Fred Soupa.

\section{BIBLIOGRAPHIE}

Bailleul, Corentin ; Senovilla Hernández, Daniel (2016) Dans l'intérêt supérieur de qui ? Enquête sur l'interprétation et l'application de l'article 3 de la Convention Internationale des Droits de l'Enfant dans les mesures prises à l'égard des mineurs isolés étrangers en France, Poitiers, Migrinter, $157 \mathrm{p}$.

Ministère de la Justice (2013(a)) Circulaire relative aux modalités de prise en charge des jeunes isolés étrangers : dispositif national de mise à l'abri, d'évaluation et d'orientation, Paris, Ministère de la Justice, $6 \mathrm{p}$.

Ministère de la Justice (2013(b)) Jeunes étrangers isolés. Évaluation de l'âge et de l'isolement. Protocole d'évaluation, Paris, Ministère de la Justice, $4 \mathrm{p}$.

Przybyl, Sarah (2016) Territoires de la migration, territoires de la protection. Parcours et expériences des mineurs isolés étrangers accueillis en France, Poitiers, Université de Poitiers, 515 p., Th. Doct : Géogr : Poitiers : 2016. 


\section{NOTES}

1. En 2016, le garde des Sceaux souhaite que l'expression de "mineur isolé étranger » ne fasse plus la spécificité de la France. Pour se rallier aux normes européennes et internationales, mais aussi pour rappeler que les mineurs relèvent avant tout de la protection de l'enfance en danger, il signale qu'il sera dorénavant question de « mineur non accompagné ». Malgré cette déclaration, l'appellation de "mineur isolé étranger » utilisée depuis une trentaine d'années reste toujours bien ancrée dans les discours et les pratiques des acteurs en lien avec ce public.

INDEX

Mots-clés : mineurs isolés, intégration sociale, art, histoire de vie

\section{AUTEURS}

\section{SARAH PRZYBYL}

Docteure en Géographie, Migrinter, UMR 7301, CNRS / Université de Poitiers sarah.przybyl@gmail.com

\section{FRED SOUPA}

Réalisateur, Association La Colline

lacolline.asso@orange.fr 